\title{
Urticaire chronique et corticothérapie générale, les liaisons dangereuses
}

\author{
Chronic urticaria and systemic corticosteroids, the dangerous links
}

\author{
E. Amsler · A. Nosbaum - Groupe urticaire de la Société française de dermatologie \\ (C) SFMU et Springer-Verlag France 2011
}

La majorité des patients se présentant dans un service d'urgence avec une poussée d'urticaire généralisée et/ou un angio-œdème va recevoir un traitement associant corticoïde et antihistaminique. Cette attitude pragmatique permet dans bien des cas de réduire l'évolution de l'urticaire aiguë tout en soulageant le malade, mais n'est confortée que par de rares études $[1,2]$. Le but de cet éditorial n'est pas tant de remettre en question cette attitude thérapeutique que d'attirer l'attention des urgentistes sur une situation où la corticothérapie peut être délétère, l'urticaire chronique (UC). Bien que fréquente, cette pathologie est souvent méconnue des médecins et des patients.

\section{Comment reconnaître une UC ?}

Le diagnostic d'UC repose sur l'interrogatoire ; par définition, c'est une urticaire évoluant depuis plus de six semaines [3]. Lorsque les poussées sont quotidiennes ou quasi quotidiennes, le diagnostic est simple, il devient plus difficile lorsqu'elles sont intermittentes. Il faut savoir rechercher à l'interrogatoire la survenue fréquente et répétée de plaques prurigineuses et fugaces d'urticaire. L'angio-œdème ou urticaire profonde est associé aux plaques d'urticaire chez $40 \%$ des UC et peut être isolé dans $10 \%$ des cas [4]. La méconnaissance du diagnostic amène les patients à des consultations multiples et répétées aux urgences par crainte de manifestations anaphylactiques graves dont le traitement d'urgence est l'adrénaline.

E. Amsler $(\bowtie)$

Service dermatologie-allergologie, hôpital Tenon,

4, rue de la Chine, F-75020 Paris, France

e-mail : emmanuelle.amsler@tnn.aphp.fr

\section{A. Nosbaum}

Inserm U 851, service d'allergologie et immunologie clinique,

CHU Lyon-Sud, hôpitaux de Lyon, université Lyon-1,

F-69495 Pierre-Bénite cedex, France

Groupe urticaire de la Société française de dermatologie (GUS)

Maison de la dermatologie, 25, rue de la Boétie,

F-75008 Paris, France

\section{Pourquoi faut-il éviter la corticothérapie générale dans l'UC ?}

- Il s'agit par définition d'une pathologie chronique, souvent prolongée. Une étude portant sur 139 urticariens chroniques a ainsi montré la persistance de la maladie dans $52 \%$ des cas à deux ans, $43 \%$ à trois ans et $14 \%$ à cinq ans [5]. Le recours répétitif à la corticothérapie par méconnaissance du diagnostic peut générer une corticodépendance et des effets secondaires non négligeables dans une pathologie cutanée, certes invalidante en termes de qualité de vie, mais bénigne ;

- la corticothérapie générale n'est pas un traitement curatif de l'UC, aucun n'étant d'ailleurs validé dans cette pathologie [6]. Le traitement symptomatique repose sur la prise continue d'antihistaminiques $\mathrm{H} 1$ qui permettent dans un grand nombre de cas de soulager suffisamment les patients. Une étude rétrospective portant sur 750 adultes atteints d'UC a ainsi montré une réponse satisfaisante à une monothérapie antihistaminique chez $88 \%$ des patients [7]. Le consensus européen de 2009 ne recommande l'usage de la corticothérapie générale dans les exacerbations sévères qu'en troisième " palier » et en cure courte de trois à sept jours après échec des anti-H1 à posologie normale puis majorée et des antileucotriènes. Cette recommandation ne repose cependant que sur un faible niveau de preuve [6] ;

- une corticothérapie répétée semble pouvoir aggraver l'UC et induire une résistance aux antihistaminiques [8]. Ainsi, dans une étude prospective ouverte portant sur 17 urticariens chroniques sévères traités par corticoïdes et résistant aux antihistaminiques, l'arrêt de toute corticothérapie a permis d'obtenir, sous anti-H1, six rémissions complètes et huit rémissions partielles à un an [8].

\section{Conclusion}

L'UC requiert, comme toute pathologie chronique, l'éducation des patients qui doivent être informés de la nécessité de 
prendre un traitement antihistaminique de longue durée. Un document explicatif à l'usage des patients a été créé pour apporter des explications simples sur cette maladie (www.sfdermato.org). Il est également important que les médecins urgentistes sachent reconnaître l'UC pour rassurer les patients, leur apporter une prise en charge adaptée et éviter le recours à la corticothérapie générale.

\section{Références}

1. Pollack CV, Romano TJ (1995) Outpatient management of acute urticaria: the role of prednisone. Ann Emerg Med 26:547-51

2. Zuberbier T, Ifflander J, Semmler C, et al (1996) Acute urticaria: clinical aspects and therapeutic responsiveness. Acta Derm Venereol 76:295-7
3. Agence nationale d'accréditation et d'évaluation en santé, Société française de dermatologie (2003) Prise en charge de l'urticaire chronique. Ann Dermatol Venereol 130(Suppl 1):S182-S92

4. Powell RJ, Du Toit GL, Siddique N, et al (2007) BSACI guidelines for the management of chronic urticaria and angio-oedema. Clin Exp Allergy 37:631-50

5. Toubi E, Kessel A, Avshovich N, et al (2004) Clinical and laboratory parameters in predicting chronic urticaria duration: a prospective study of 139 patients. Allergy 59:869-73

6. Zuberbier T, Asero R, Bindslev-Jensen C, et al (2009) EAACI/GA (2) LEN/EDF/WAO guideline: management of urticaria. Allergy 64:1427-43

7. Asero R, Tedeschi A (2010) Usefulness of a short course of oral prednisone in antihistamine-resistant chronic urticaria: a retrospective analysis. J Investig Allergol Clin Immunol 20:386-90

8. Augey F, Guillot-Pouget I, Gunera-Saad N, et al (2008) Effet de l'arrêt des corticoïdes au cours de l'urticaire chronique : étude prospective de 17 cas. Ann Dermatol Venereol 135:21-5 